\title{
Electric charge quantization in a chiral bilepton gauge model
}

\author{
C. A. de S. Pires and O. P. Ravinez \\ Instituto de Física Teórica, Universidade Estadual Paulista, Rua Pamplona 145, 01405-900 São Paulo, São Paulo, Brazil
}

(Received 30 January 1998; published 6 July 1998)

\begin{abstract}
In the context of the standard model the quantization of the electric charge occurs only family by family. When we consider the three families together with massless neutrinos the electric charge is not quantized any more. Here we show that a chiral bilepton gauge model based on the gauge group $\mathrm{SU}(3)_{C} \otimes \mathrm{SU}(3)_{L}$ $\otimes \mathrm{U}(1)_{N}$ explains the quantization of the electric charge when we take into account the three families of fermions. This result does not depend on the neutrino masses. Charge quantization occurs whether the neutrinos are massless or Dirac or Majorana massive fields. [S0556-2821(98)02115-8]

PACS number(s): 12.60.Cn, 12.90.+b
\end{abstract}

\section{INTRODUCTION}

The reason why the electric charges of fundamental particles appear only in discrete units is still an open question. Over the years some proposals have appeared intending to explain it. The first proposal was given by Dirac [1] through the postulate of the magnetic monopoles. The second proposal comes from the grand unification theories (GUT's) through its group structure [2]. But the GUT's are ruled out and magnetic monopoles still have not been detected.

Recently the quantization of the electric charge (QECH) has been analyzed within gauge models that contain the $\mathrm{U}(1)$ factor in its gauge group [3-5]. The approach given here relates the $\mathrm{U}(1)$ charges of the fermions and Higgs bosons of the model through classical and quantum constraints in such a manner that it leads to the QECH. The classical constraints imply that the Lagrangian of the model be invariant by the gauge group; the quantum ones imply that the model be free from anomalies $[3,4,6]$.

By analyzing the QECH in the standard model (SM), whose gauge group is $G_{S M}=\mathrm{SU}(3)_{C} \otimes \mathrm{SU}(2)_{L} \otimes \mathrm{U}(1)_{Y}$, several authors showed that the SM with one family contains the QECH $[3-5,7,8]$. Nevertheless, when we increase the number of families to three the effect of dequantization occurs $[3,8,9]$. To understand this we need to see that in the SM with three families an independent anomaly free global $\mathrm{U}(1)_{Y_{1}}$ symmetry arises $[3,4]$. By independent we mean that the $\mathrm{U}(1)_{Y_{1}}$ is independent of the gauge symmetry of the SM. By anomaly free we mean that the $\mathrm{U}(1)_{Y_{1}} G_{S M}^{2}$ and $\mathrm{U}(1)_{Y_{1}}^{3}$ anomalies are canceled [3]. This kind of symmetry is also called hidden symmetry. It creates an arbitrariness in the definition of the electric charge operator since $Y$ and $Y$ $+\alpha Y_{1}$ are equally good choices for the gauge $\mathrm{U}(1)$ in the gauge group of the standard model $[3,4]$.

We show, using this approach, that in a chiral bilepton gauge model, proposed some years ago by Pisano, Pleitez, and Frampton [10], based on the symmetry gauge group $G_{331}=\mathrm{SU}(3)_{C} \otimes \mathrm{SU}(3)_{L} \otimes \mathrm{U}(1)_{N}$, there is the $\mathrm{QECH}$ through classical and quantum constraints independently if neutrinos are massless or not. This model embeds the SM; it adds new physics with no hidden symmetries, the leptons come in the $\mathrm{SU}(3)_{L}$ representation and it has three nontrivial anomaly cancellations [11].
This paper is organized as follows. In Sec. II we analyze the QECH in the SM with one and three families showing the hidden symmetries that lead to the effect of dequantization. In Sec. III we extend the analysis to the chiral bilepton gauge model and we obtain the quantization of the electric charge through classical and quantum constraints and in Sec. IV we summarize our conclusions.

\section{THE QUANTIZATION IN THE STANDARD MODEL}

\section{A. The standard model with one family}

The electric charge operator in the SM can be defined in a general form as

$$
Q=T_{3}+b Y
$$

where $b$ is an unknown parameter. For nonvanishing fermion masses we must introduce a Higgs doublet $\phi \sim\left(\mathbf{1}, \mathbf{2}, Y_{\phi}\right)$ that acquires a vacuum expectation value

$$
\langle\phi\rangle_{0} \sim\left(\begin{array}{l}
0 \\
v
\end{array}\right) .
$$

Since we want the operator $Q$ unbroken, $Q\langle\phi\rangle_{0}$ must be zero. With this condition we find $b=\frac{1}{2} Y_{\phi}$. So the electric charge operator takes the form

$$
Q=T_{3}+\frac{Y}{2 Y_{\phi}} .
$$

Then, the problem of the quantization of the electric charge turns into writing all hypercharges as functions of the Higgs hypercharge $Y_{\phi}$. To achieve this we use the classical and quantum constraints. The only sector in the Lagrangian able to give information about the hypercharges through classical constraints is the Yukawa one, while the information about the hypercharges through quantum constraints comes from the three nontrivial anomaly cancellations $\left[\mathrm{U}(1)_{Y}\right]^{3}$, $\left[\mathrm{SU}(2)_{L}\right]^{2} \mathrm{U}(1)_{Y}$, and $\left[\mathrm{SU}(3)_{C}\right]^{2} \mathrm{U}(1)_{Y}$.

In the SM the quarks and leptons come in the following representations:

$$
L_{L}=\left(\begin{array}{c}
\nu_{e} \\
e
\end{array}\right)_{L} \sim\left(\mathbf{1}, 2, Y_{l}\right), \quad e_{R} \sim\left(\mathbf{1}, \mathbf{1}, Y_{e}\right),
$$




$$
\begin{gathered}
Q_{L}=\left(\begin{array}{l}
u \\
d
\end{array}\right)_{L} \sim\left(\mathbf{3}, \mathbf{2}, Y_{q}\right), \quad u_{R} \sim\left(\mathbf{3}, \mathbf{1}, Y_{u}\right), \\
d_{R} \sim\left(\mathbf{3}, \mathbf{1}, Y_{d}\right),
\end{gathered}
$$

with the Yukawa interaction being

$$
-\mathcal{L}^{Y}=g_{1}^{l} \bar{L}_{L} \phi e_{R}+g_{3}^{d} \bar{Q}_{L} \phi d_{R}+g_{2}^{u} \bar{Q}_{L} \widetilde{\phi} u_{R}+\text { H.c. }
$$

This Lagrangian, being $\mathrm{U}(1)_{Y}$ gauge invariant, implies that

$$
Y_{e}=Y_{l}-Y_{\phi}, \quad Y_{u}=Y_{q}+Y_{\phi}, \quad Y_{d}=Y_{q}-Y_{\phi} .
$$

After this only two nontrivial anomaly constraints remain,

$$
\begin{gathered}
{\left[\mathrm{SU}(2)_{L}\right]^{2} \mathrm{U}(1)_{Y} \Rightarrow Y_{q}=-\frac{1}{3} Y_{l},} \\
{\left[\mathrm{U}(1)_{Y}\right]_{Y}^{3} \Rightarrow Y_{l}=-Y_{\phi} .}
\end{gathered}
$$

Equations (6) and Eqs. (7) leave all fermion hypercharges as functions of the Higgs one,

$$
\begin{gathered}
Y_{l}=-Y_{\phi}, \quad Y_{e}=-2 Y_{\phi}, \quad Y_{q}=\frac{1}{3} Y_{\phi}, \\
Y_{u}=\frac{4}{3} Y_{\phi}, \quad Y_{d}=-\frac{2}{3} Y_{\phi} .
\end{gathered}
$$

Substituting the above results into Eq. (3), we obtain the quantization of the electric charge with the correct electric charges for leptons and quarks:

$$
Q_{\nu}=0, \quad Q_{e}=-1, \quad Q_{u}=\frac{2}{3}, \quad \text { and } \quad Q_{d}=-\frac{1}{3} .
$$

Next if we admit a right-handed neutrino with Dirac mass term and we attribute the hypercharge $Y_{\nu}$ to it we find from the Yukawa term $\bar{L}_{L} \widetilde{\phi} \nu_{R}$ that $Y_{\nu}=Y_{l}+Y_{\phi}$, but now we only have one nontrivial anomaly constraint $\left[\mathrm{SU}(2)_{L}\right]^{2} \mathrm{U}(1)_{Y}$. So we have three free parameters $Y_{l}, Y_{q}$, and $Y_{\phi}$ from classical constraints and only one equation from quantum constraints. This prevents us from leaving all the hypercharges as functions of only the Higgs hypercharge. This is the dequantization effect $[3,4]$. The explanation is that the SM with Dirac massive neutrinos present, besides the barionic $(B)$ and leptonic $(L)$ global symmetries, the $B-L$ global symmetry. The $B$ and $L$ symmetries are not free from anomalies. So they are not hidden symmetries. Nevertheless, the $B-L$ symmetry is free from anomalies. Then it is a hidden symmetry which superposes to the hypercharge one and which obstructs us from knowing if the $\mathrm{U}(1)_{Y}$ factor in the electric charge operator is due to $Y$ or to the superposition $Y+\alpha(B-L)[3,4]$. Now if we, instead of Dirac neutrinos, have a Majorana one with the mass-term $\nu_{R}^{T} C^{-1} \nu_{R}$ that breaks the $B-L$ global symmetry, we restore the quantization condition [5].

\section{B. The standard model with three families}

With three families the representation content is

$$
\begin{gathered}
L_{a L}=\left(\begin{array}{c}
\nu_{a} \\
e_{a}
\end{array}\right)_{L} \sim\left(\mathbf{1}, 2, Y_{l_{a}}\right), \quad e_{a R} \sim\left(\mathbf{1}, 1, Y_{e_{a}}\right), \\
Q_{a L}=\left(\begin{array}{c}
u_{a} \\
d_{a}
\end{array}\right)_{L} \sim\left(\mathbf{3}, 2, Y_{q_{a}}\right), \quad u_{a R} \sim\left(\mathbf{3}, 1, Y_{u_{a}}\right), \\
d_{a R} \sim\left(\mathbf{3}, 1, Y_{d_{a}}\right),
\end{gathered}
$$

with the Yukawa interaction being

$$
-\mathcal{L}^{Y}=g_{a a}^{l} \bar{L}_{a L} \phi e_{a R}+g_{a b}^{d} \bar{Q}_{a L} \phi d_{b R}+g_{a b}^{u} \bar{Q}_{a L} \widetilde{\phi} u_{b R}+\text { H.c. }
$$

where $a, b=1,2,3$. have

In order to be this Lagrangian $\mathrm{U}(1)_{Y}$ gauge invariant we

$$
\begin{aligned}
& Y_{e_{a}}=Y_{l_{a}}-Y_{\phi}, \\
& Y_{q_{a}}=Y_{q}, \quad Y_{u_{a}}=Y_{u}, \quad Y_{d_{a}}=Y_{d},
\end{aligned}
$$

with

$$
Y_{u}=Y_{q}+Y_{\phi}, \quad Y_{d}=Y_{q}-Y_{\phi} .
$$

After that we have only two nontrivial anomaly constraints,

$$
\left[\mathrm{SU}(2)_{L}\right]^{2} \mathrm{U}(1)_{Y} \text { and }\left[\mathrm{U}(1)_{Y}\right]_{Y}^{3} \text {. }
$$

So we have five free parameters from the classical constraints, Eq. (12), and only two equations from the quantum constraints, Eq. (13). This prevents us from obtaining the QECH. This is again the effect of dequantization. The hidden symmetry here is $\mathrm{U}(1)_{L}$, with $L$ being one of the quantum numbers: $L=L_{e}-L_{\mu}, L_{e}-L_{\tau}, L_{\mu}-L_{\tau}[3]$.

To restore the QECH in the SM with three families we need to introduce either right-handed neutrinos with Majorana mass terms [3,8], or another Higgs doublet [8], or some neutral fermions [9].

\section{THE QUANTIZATION IN A CHIRAL BILEPTON GAUGE MODEL}

In a chiral bilepton gauge model presented in the Introduction the electric charge operator can be defined in a general form as

$$
Q=\frac{1}{2}\left(\lambda_{3}-\sqrt{3} \lambda_{8}\right)+b N,
$$

with $N$ being the operator generator of the group $\mathrm{U}(1)_{N} ; \lambda_{3}$ and $\lambda_{8}$ being the two diagonal Gell-Mann matrices.

In order to break the symmetry spontaneously and to give mass to the fermions, three Higgs triplets and one Higgs sextet doublets are introduced [10-12], 


$$
\begin{aligned}
\eta \sim\left(1,3, N_{\eta}\right), & \rho \sim\left(1,3, N_{\rho}\right), \quad \chi \sim\left(1,3, N_{\chi}\right), \\
& S \sim\left(1,6, N_{S}\right),
\end{aligned}
$$

with the corresponding $\mathrm{U}(1)_{N}$ charges as unknown parameters.

To generate mass correctly, those Higgs boson must acquire the following vacuum expectation values [10-12]:

$$
\begin{gathered}
\langle\eta\rangle_{0} \sim\left(\begin{array}{c}
v_{\eta} \\
0 \\
0
\end{array}\right), \quad\langle\rho\rangle_{0} \sim\left(\begin{array}{c}
0 \\
v_{\rho} \\
0
\end{array}\right), \\
\langle\chi\rangle_{0} \sim\left(\begin{array}{c}
0 \\
0 \\
v_{\chi}
\end{array}\right), \quad\langle S\rangle_{0} \sim\left(\begin{array}{ccc}
0 & 0 & 0 \\
0 & 0 & v^{\prime} \\
0 & v^{\prime} & 0
\end{array}\right) .
\end{gathered}
$$

With the requirement that the charge operator must annihilate the vacuum, we obtain the following relations:

$$
N_{\eta}=0, \quad b=\frac{1}{N_{\rho}}, \quad N_{\chi}=-N_{\rho}, \quad N_{S}=0 .
$$

With these results we can write the electric charge operator in the following form:

$$
Q=I_{3}+\frac{Y}{2}
$$

with the hypercharge $Y$ being

$$
\frac{Y}{2}=I_{8}+\frac{N}{N_{\rho}}
$$

where $I_{3}=\frac{1}{2} \lambda_{3}$ and $I_{8}=-\sqrt{3} / 2 \lambda_{8}$.

Now the problem of the quantization of the electric charge consists of writing all the $\mathrm{U}(1)_{N}$ charges as functions of $N_{\rho}$. We achieve this in the same way as we did for the hypercharges in the SM, that is, by making use of the classical and quantum constraints. The only sector in the Lagrangian able to give information about the $\mathrm{U}(1)_{N}$ charges through classical constraints is the Yukawa one, while the information about the $\mathrm{U}(1)_{N}$ charges through quantum constraints comes from the three nontrivial anomaly cancellations, $\left[\mathrm{U}\left(1_{N}\right)\right]^{3},\left[\mathrm{SU}(3)_{C}\right]^{2} \mathrm{U}(1)_{N}$, and $\left[\mathrm{SU}(3)_{L}\right]^{2} \mathrm{U}(1)_{N}$.

The leptons in the model come in the $\mathrm{SU}(3)$ representation

$$
L_{a L}=\left(\begin{array}{c}
\nu_{a} \\
e_{a} \\
e_{a}^{c}
\end{array}\right)_{L} \sim\left(\mathbf{1}, \mathbf{3}, N_{l_{a}}\right),
$$

with $a=1,2,3$. The right-handed charged leptons enter the model through charge conjugation, i.e., there are no lepton singlets.

The quarks belong to $\mathrm{SU}(3)$ and $\mathrm{U}(1)$ representations and one family comes in triplets and the other ones in antitriplets:

$$
\begin{gathered}
Q_{1 L}=\left(\begin{array}{c}
u_{1} \\
d_{1} \\
J_{1}
\end{array}\right)_{L} \sim\left(\mathbf{3}, \mathbf{3}, N_{q_{1}}\right), \\
u_{1 R} \sim\left(\mathbf{3}, \mathbf{1}, N_{u_{1}}\right), \quad d_{1 R} \sim\left(\mathbf{3}, \mathbf{1}, N_{d_{1}}\right), \quad J_{R_{1}} \sim\left(\mathbf{3}, \mathbf{1}, N_{J_{1}}\right) . \\
Q_{i L}=\left(\begin{array}{c}
d_{i} \\
u_{i} \\
J_{i}
\end{array}\right)_{L} \sim\left(\mathbf{3}, \mathbf{3}^{*}, N_{q_{i}}\right), \\
d_{i R} \sim\left(\mathbf{3}, \mathbf{1}, N_{d_{i}}\right), \quad u_{i R} \sim\left(\mathbf{3}, \mathbf{1}, N_{u_{i}}\right), \quad J_{i R} \sim\left(\mathbf{3}, \mathbf{1}, N_{J_{i}}\right),
\end{gathered}
$$

where $i=2,3$. The quarks $u e d$ are the usual ones with $J$ being the exotic quarks. Now we are ready to obtain the QECH.

In order to obtain relations among these $\mathrm{U}(1)_{N}$ charges through the classical constraints, we again use the Yukawa Lagrangian sector [12]

$$
\begin{aligned}
-\mathcal{L}_{Y}= & \frac{1}{2} G_{a b} \bar{L}_{a L}^{c} * L_{b L}+\lambda_{1} \bar{Q}_{1 L} J_{1 R} \chi+\lambda_{i j} \bar{Q}_{i L} J_{j R} \chi^{*} \\
& +\lambda_{1 a}^{\prime} \bar{Q}_{1 L} d_{a R} \rho+\lambda_{i a}^{\prime} \bar{Q}_{i L} u_{a R} \rho^{*}+\lambda_{1 a}^{\prime \prime} \bar{Q}_{1 L} u_{a R} \eta \\
& +\lambda_{i a}^{\prime \prime} \bar{Q}_{i L} d_{a R} \eta^{*}+\text { H.c. }
\end{aligned}
$$

where $a, b=1,2,3$ and $i, j=2,3 . \chi^{*}, \rho^{*}$ and $\eta^{*}$ are antitriplets, while $S^{*}$ is an antisextet.

The main point here is the leptonic sector of the Yukawa Lagrangian. Its framework provides the $\mathrm{U}(1)_{N}$ charges of the leptons in a direct manner, that is, the $\mathrm{U}(1)_{N}$ gauge invariance of this term implies

$$
N_{l_{1}}=N_{l_{2}}=N_{l_{3}}=0 \text {. }
$$

The $\mathrm{U}(1)_{N}$ gauge invariance of the quark sectors of the Yukawa Lagrangian implies

$$
\begin{aligned}
& N_{u_{1}}=N_{u_{2}}=N_{u_{3}}=N_{u}, \\
& N_{d_{1}}=N_{d_{2}}=N_{d_{3}}=N_{d}, \\
& N_{q_{2}}=N_{q_{3}}=N_{q}, \\
& N_{J_{2}}=N_{J_{3}}=N_{J},
\end{aligned}
$$

and 


$$
\begin{aligned}
& N_{J}=N_{q}-N_{\rho}, \\
& N_{d}=N_{q}, \\
& N_{u}=N_{q}+N_{\rho}, \\
& N_{J_{1}}=N_{q}+2 N_{\rho}, \\
& N_{q_{1}}=N_{q}+N_{\rho} .
\end{aligned}
$$

After that we have only one nontrivial anomaly cancellation [13]

$$
\left[\mathrm{SU}(3)_{L}\right]^{2} \mathrm{U}(1)_{N} \Rightarrow N_{q_{1}}+2 N_{q}=0
$$

From the last term in Eqs. (25) and (26) we find

$$
N_{q}=-\frac{1}{3} N_{\rho},
$$

which leads to the following relations among the $\mathrm{U}(1)_{N}$ charges:

$$
\begin{aligned}
N_{q_{1}}=\frac{2}{3} N_{\rho}, & N_{q}=-\frac{1}{3} N_{\rho}, \\
N_{d}=-\frac{1}{3} N_{\rho}, & N_{u}=\frac{2}{3} N_{\rho}, \\
N_{J 1}=\frac{5}{3} N_{\rho}, & N_{J}=-\frac{4}{3} N_{\rho} .
\end{aligned}
$$

This result allows us, together with Eqs. (24) and (19) to find [when we replace the value of the corresponding $\mathrm{U}(1)_{N}$ charges] the hypercharges of all fermions. Substituting the hypercharges into Eq. (18) we find the quantization of the electric charge with the correct electric charges for leptons and quarks,

$$
\begin{aligned}
Q_{\nu_{e}, \nu_{\mu}, \nu_{\tau}} & =0, \\
Q_{e, \mu, \tau} & =Q=-1, \\
Q_{u} & =+\frac{2}{3}, \\
Q_{d} & =-\frac{1}{3}, \\
Q_{J 1} & =+\frac{5}{3}, \\
Q_{J 2, J 3} & =Q_{J}=-\frac{4}{3} .
\end{aligned}
$$

Then, we showed that the chiral bilepton gauge model based on a semisimple Lie group $G_{331}$ contains in its framework the quantization of the electric charge when we take into account the three families of fermions with massless neutrinos.

We finish this section making a short analysis of the extensions of this model in order to consider massive neutrinos. For Majorana neutrinos we only need to use the following vacuum expectation value for the sextet [12]:

$$
\langle S\rangle_{0} \sim\left(\begin{array}{ccc}
v & 0 & 0 \\
0 & 0 & v^{\prime} \\
0 & v^{\prime} & 0
\end{array}\right)
$$

This conserves the structure of the leptonic sector, leading also to the quantization condition. Now if we want a Dirac neutrino we need to add the following term $G_{a b}^{\prime} \bar{L}_{a L} \eta \nu_{a R}$ to the Lagrangian $\mathcal{L}_{Y}$ in Eq. (23) with $\nu_{a R} \sim\left(\mathbf{1}, \mathbf{1}, N_{\nu_{a}}\right)$. By gauge invariance we find $N_{\nu_{a}}=0$. This result maintains the quantization condition. All of this shows that this model is interesting in looking for new physics.

\section{CONCLUSIONS}

In summary, we have extended the recent approach of the electric charge quantization problem in gauge models that contain an explicit $\mathrm{U}(1)$ gauge group to the case of one $G_{331}$ model. First we showed through classical and quantum constraints that the standard model with one family explains the quantization of the electric charge, while with three families and massless neutrinos it no longer explains the charge quantization. We discussed the reasons for the above result and we also showed that by adding neutrinos with a Majorana mass to the standard model we restored the condition of the electric charge quantization. This happens because Majorana neutrinos break the $\mathrm{U}(1)_{L}$ hidden symmetry that arises when we consider the standard model with three families.

The central part of this work analyzed the question of the quantization of the electric charge in a $G_{331}$ model and the main result in this work is the following: the QECH through classical and quantum constraints occurs in the $G_{331}$ model when the three families are taken together even if neutrinos are massless or not. If they are massive the QECH does not depend on the nature of the neutrino fields, i.e., it does not matter if they are Dirac or Majorana fermions.

\section{ACKNOWLEDGMENTS}

We thank J.C. Montero and V. Pleitez for the incentive and critical suggestions and also thank M.C. Tijero for reading the manuscript. This work was supported by the Conselho Nacional de Desenvolvimento Científico e Tecnológico (CNPq) (O.P.R) and the Coordenação de Aperfeiçoamento de Pessoal de Nível Superior (CAPES) (C.A.S.P). 
[1] P. A. M. Dirac, Proc. R. Soc. London A133, 60 (1931).

[2] P. Langacker, Phys. Rep. 72, 185 (1981).

[3] R. Foot, G. C. Joshi, H. Lee, and R. R. Volkas, Mod. Phys. Lett. A 5, 2721 (1990); R. Foot, ibid. 6, 527 (1991); R. Foot, H. Lee, and R. R. Volkas, J. Phys. G 19, 269 (1993).

[4] K. S. Babu, and R. N. Mohapatra, Phys. Rev. D. 41, 271 (1990).

[5] K. S. Babu and R. N. Mohapatra, Phys. Rev. Lett. 63, 938 (1989).

[6] S. Adler, Phys. Rev. 177, 2426 (1969); J. S. Bell and R. Jackiw, Nuovo Cimento A 60, 49 (1969).

[7] S. Rudaz, Phys. Rev. D 41, 2619 (1990); C. Q. Geng and R. E.
Marshak, ibid. 39, 693 (1989); E. Golowich and P. B. Pal, ibid. 41, 3757 (1990).

[8] J. Sladkowski and M. Zralek, Phys. Rev. D 45, 1701 (1992).

[9] M. Nowakowski and A. Pilaftsis, Phys. Rev. D 48, 259 (1993).

[10] F. Pisano and V. Pleitez, Phys. Rev. D 46, 410 (1992); H. Frampton, Phys. Rev. Lett. 69, 2889 (1992).

[11] F. Pisano, V. Pleitez, and M. D. Tonasse, in "Flavor chiral extensions for the standard model,' Report No. IFT-P.043/97 (unpublished).

[12] R. Foot, O. F. Hernández, F. Pisano, and V. Pleitez, Phys. Rev. D 47, 4158 (1993).

[13] F. Pisano, Mod. Phys. Lett. A 11, 2539 (1996). 